\title{
Arbeitgebermaßnahmen gegen Betriebsräte: Angriffe auf die betriebliche Mitbestimmung
}

Das bundesdeutsche System der industriellen Beziehungen gilt im internationalen Vergleich als betont sozialpartnerschaftlich ausgerichtet. Dezidiert gewerkschaftsfeindliches Vorgehen seitens der Unternehmen und des Managements wird hier üblicherweise nicht verortet. Die Behinderung von Betriebsratswahlen ist verboten und unter Strafe gestellt. Dennoch häufen sich in den letzten Jahren zunehmend Berichte, dass genau dies geschieht. Tatsächlich zeigen die Ergebnisse einer vom Wirtschafts- und Sozialwissenschaftlichen Institut (WSI) in Kooperation mit betroffenen DGB-Gewerkschaften durchgeführten Studie, dass Manager teilweise massiv und hartnäckig die Errichtung von Betriebsräten zu verhindern suchen. Der Artikel stellt die bisher vorliegenden Forschungsergebnisse vor und zeigt, dass besonders häufig inhabergeführte Unternehmen sich schwer tun, die betriebliche Mitbestimmung zu akzeptieren.

MARTIN BEHRENS, HEINER DRIBBUSCH

\section{Einleitung}

Im Frühjahr 2012 erklärte die Drogeriekette Schlecker ihre Insolvenz. Neben einem als überholt angesehenen Geschäftsmodell war es das negative Image der Firma als eines der gewerkschafts- und mitbestimmungsfeindlichsten Unternehmen Deutschlands, das nachhaltig das Geschäft schädigte (Küppers 2012). Im Zuge der Insolvenz verloren 24.000 überwiegend weibliche Beschäftigte ihre Arbeit. Die besondere Tragik dieses Ereignisses lag darin, dass die SchleckerFrauen gerade wegen ihres couragierten Auftretens große öffentliche Sympathien genossen. In einer der bedeutendsten Kampagnen im Einzelhandel (Huhn 2001) und in vielen mühsamen Auseinandersetzungen (Bormann 2007) war die Bildung von Betriebsräten und schließlich auch eine Tarifbindung durchgesetzt worden. Auch wenn Schlecker als besonders spektakuläres Beispiel für den Widerstand von Unternehmen gegen die Errichtung von Betriebsräten stand, so war und ist es nicht der einzige öffentlich gewordene Fall massiver Behinderung der betrieblichen Mitbestimmung. Lidl ist ein weiteres prominentes Beispiel, doch wurden in den letzten Jahren auch zahlreiche Fälle außerhalb des Einzelhandels bekannt.

Dies mag für Außenstehende zunächst insofern erstaunlich scheinen, als die deutschen Arbeitsbeziehungen in der Regel nicht mit Phänomenen wie massiver Mitbestimmungs- und Gewerkschaftsfeindlichkeit in Verbindung ge- bracht werden. „Union busting“ - also bewusste arbeitgeberseitige Initiativen zur Zerschlagung der Gewerkschaften - gelten als ein Phänomen der angelsächsischen Länder und hier insbesondere der Vereinigten Staaten (Logan 2006). Dieses oberflächliche Bild täuscht. Zwar gibt es in Deutschland anders als in den angelsächsischen Ländern kein förmliches Anerkennungsverfahren, in dem zunächst einmal für jedes Unternehmen geklärt werden muss, ob die betreffende Gewerkschaft überhaupt verhandlungsberechtigt ist. Jedoch gilt auch in Deutschland, dass gewerkschaftliche Organisierung in die Machtverteilung zwischen Beschäftigten und Unternehmen eingreift und deshalb im wörtlichen Sinne umstritten ist.

Aus der Perspektive der Gewerkschaft lässt sich der Aufbau einer betrieblicher Verankerung modellhaft als zweistufiger, organisierter Prozess aus betrieblicher Organisierung und individueller Mitgliederwerbung beschreiben (Haas 2000; Dribbusch 2003). Die erste zu überwindende Hürde - der Aufbau gewerkschaftlicher Vertretungsstrukturen - fällt in Deutschland in der Regel mit der Errichtung von Betriebsräten zusammen. Nun ist der Betriebsrat formal keine gewerkschaftliche Struktur und die einzelnen Betriebsratsmitglieder können eine durchaus unterschiedliche Nähe zur Gewerkschaft pflegen (Schmidt/Trinczek 1999). Doch gilt, dass in Betrieben ohne Betriebsrat in der Regel auch keine nennenswerte gewerkschaftliche Präsenz vorhanden ist (vgl. Dribbusch 2003). Gewerkschaftlich organisierte Betriebsräte helfen, die gewerkschaftliche Mitgliederbasis und damit 
die Grundlage für die Durchsetzung tarifpolitischer Forderungen zu erneuern (Behrens 2009) und zu erweitern und begrenzen darüber hinaus durch ihre Mitbestimmungsaktivitäten die freie Gestaltungsmacht des betrieblichen Managements. Immerhin drei Viertel der 2010 gewählten Betriebsratsmitglieder gehörten einer ersten Auswertung zufolge einer DGB-Gewerkschaft an (Greifenstein et al. 2011). Auseinandersetzungen um die Errichtung von Betriebsräten sind insofern ein Pendant zu den Anerkennungskonflikten in angelsächsischen Ländern. Beide Male geht es darum, zu verhindern, dass sich eine Gewerkschaft im Betrieb etabliert.

In der Mehrzahl der Betriebe, die über einen gewählten Betriebsrat verfügen, bleibt diese institutionell verankerte Machtteilung unwidersprochen. Sie wird in einem System der kooperativen Arbeitsbeziehungen toleriert, wenn nicht sogar geschätzt. Wie wir in der Folge zeigen werden, kann von einer solchen Akzeptanz im Bereich der betriebsratslosen Betriebe nicht die Rede sein.

In den folgenden Abschnitten gehen wir daher der Frage nach, wie verbreitet Maßnahmen des Managements gegen die betriebliche Mitbestimmung in Deutschland sind. Unter Rückgriff auf Daten der WSI-Befragung hauptamtlicher Gewerkschafter 2012 analysieren wir darüber hinaus, inwieweit sich die Häufigkeit und Struktur solchen Managementverhaltens in betriebsratslosen Betrieben von der Situation in Betrieben mit bereits etablierten Betriebsräten unterscheidet.

Wir beginnen mit einer Sondierung des Terrains. Unter Bezug auf vorliegende Arbeiten gehen wir dann der Frage nach den Motivationen für die Behinderung der Betriebsratsgründung nach (Abschnitt 2). Dann stellen wir zunächst unsere Erhebung vor (3), bevor wir uns in zwei Schritten den Managementversuchen zur Verhinderung der Betriebsratswahl (4) sowie Maßnahmen gegen gewählte Betriebsratsgremien (5) zuwenden. Am Ende ziehen wir ein kurzes Fazit (6).

\section{Managementopposition: Handeln in der Grauzone}

Aktive Opposition gegen die betriebliche Mitbestimmung seitens des Managements lässt sich auf zwei Ebenen beobachten: der Versuch, die Gründung eines Betriebsrates zu vereiteln, sowie Maßnahmen, gewählte Betriebsratsgremien in ihrer Existenz oder Arbeitsfähigkeit zu bedrohen. Inwieweit Unternehmen die Errichtung von Betriebsräten behindern können, hängt unter anderem von den gesetzlichen Rahmenbedingungen sowie deren Durchsetzung ab.

Die Bildung von Betriebsräten wird in Deutschland durch das Betriebsverfassungsgesetz (BetrVG) geregelt. Dieses bestimmt in $\$ 20$ sehr deutlich: „Niemand darf die Wahl des Betriebsrats behindern. “ Und weiter: „Niemand darf die Wahl des Betriebsrats durch Zufügung oder Androhung von Nachteilen oder durch Gewährung oder Versprechen von Vorteilen beeinflussen. “Verstöße hiergegen stellen nach $\$ 119$ BetrVG einen Straftatbestand dar. Grundvoraussetzung für die Wahl eines Betriebsrates ist, dass der betreffende Betrieb mindestens fünf Beschäftigte hat. Gemessen an den Möglichkeiten sind Betriebsräte jedoch eine Ausnahmeerscheinung.

2012 verfügten in Westdeutschland lediglich $9 \%$ aller infrage kommenden Betriebe mit mehr als fünf Beschäftigten über einen Betriebsrat. Dass es sich dabei vor allem um größere Betriebe handelt, wird deutlich, wenn wir sehen, dass diese Minderheit an Betrieben immerhin $43 \%$ aller infrage kommenden Beschäftigten repräsentierte. In Ostdeutschland sieht es kaum anders aus: Hier verfügen ebenfalls $9 \%$ der betriebsratsfähigen Betriebe über ein solches Gremium; da es dort weniger sehr große Betriebe gibt, repräsentierten diese jedoch nur $36 \%$ der Beschäftigten (Ellguth/Kohaut 2013, S. 285).

Es soll hier allerdings nicht der Eindruck erweckt werden, jeder betriebsratslose Betrieb sei Resultat entsprechender Abwehrreaktionen des Managements bzw. der Betriebsinhaber. Wir gehen davon aus, dass eine bedeutende Anzahl von Betrieben deshalb keinen Betriebsrat hat, weil weder die Gewerkschaft noch die Beschäftigten einen Versuch unternommen haben, einen solchen zu installieren. Hierin drückt sich bereits eine Schwierigkeit bei der Erhebung betriebsratsfeindlichen Verhaltens aus: Während also eine Unschärfe darin besteht, die einfache Abwesenheit von Betriebsräten monokausal als Produkt von Managementopposition $\mathrm{zu}$ interpretieren und somit falsch zu deuten, besteht auf der anderen Seite auch die erhebliche Gefahr, sehr effektive, aber weniger sichtbare oder „flagrante“ Managementmaßnahmen zu bagatellisieren. So wissen wir aus einer Reihe von Studien (Dribbusch 2003; Bormann 2007), dass Arbeitgeber auch jenseits von offen aggressiver Gewerkschafts- und Mitbestimmungsfeindlichkeit über ein großes Repertoire eher subtiler Möglichkeiten verfügen, die entsprechenden Adressaten wissen zu lassen, dass sie im Betrieb unerwünscht sind. Die Spannweite und Auswirkungen dieser Taktiken sind jedoch nur schwer empirisch zu messen (vgl. Ebbinghaus/Visser 1997, S. 11). Schon allein aus Gründen der empirischen Durchführbarkeit werden wir uns in der Folge deshalb auf Fälle der manifesten Behinderung der Betriebsratsarbeit konzentrieren.

Die Motivationen für solches Arbeitgeberverhalten erweisen sich, folgt man der bislang veröffentlichten Literatur, als durchaus vielschichtig. Zwar erhärten sich mittlerweile Befunde, wonach Betriebsräte neutrale, wenn nicht sogar positive Auswirkungen auf den wirtschaftlichen Erfolg der Betriebe haben (Jirjahn 2011), jedoch bedeutet dies nicht notwendigerweise, dass Arbeitgeber in Gänze ihren Widerstand gegen die Teilhabe der Beschäftigten und ihrer Vertretungen aufgeben würden. So fanden beispielsweise Böhm und Lücking (2006, S. 113) in ihrer Studie über Manager in Betrieben ohne Betriebsrat, dass sich Vertreter des 
Managements häufig distanziert gegenüber der betrieblichen Mitbestimmung positionierten und in einigen Interviews sogar „heftige Ablehnung“ gegenüber Betriebsräten äußerten (ebd., S. 108). Die Autoren identifizierten drei Grundhaltungen, die letztlich dazu führen, dass Arbeitgeber Betriebsräte als unproduktive Institutionen oder gar als Bedrohung wahrnehmen. Eine erste Grundhaltung geht davon aus, dass das Management der bessere Problemlöser sei. Manager meinen, selbst viel besser als ein Betriebsrat in der Lage zu sein, auftretende betriebliche Probleme zu lösen. Aus dieser Perspektive erweist sich der Betriebsrat als schlichtweg überflüssig. Gemäß einer zweiten Einstellung wird der Betriebsrat als eine Gefahr für den Betriebsfrieden angesehen, weil er letztlich nur egoistische, ideologisch motivierte Ziele verfolge. Schließlich, so eine dritte Managementperspektive, seien Betriebsräte zu teuer. Kosten würden zum einen durch die direkten Ausgaben für die Interessenvertretungen entstehen, aber auch durch die notwendigen Verhandlungsprozesse. Letztlich lassen sich diese Einstellungen in zwei große Komplexe bündeln: zum einen eher traditionell-autoritäre Vorstellungen des Managements, zum anderen aber auch eher moderne Konzepte, die selektiv auf Aspekte des Human Resource Managements zurückgreifen.

Auseinandersetzungen zwischen engagierten Beschäftigten und Management haben sehr rasch eine dezidiert persönliche Komponente. Dies gilt insbesondere für Konflikte um die Errichtung von Betriebsräten, die im höheren Maße personalisiert sind als beispielsweise tarifpolitische Auseinandersetzungen, in denen intermediär eine dritte Partei, nämlich die Gewerkschaft, involviert ist. Betriebsratskandidatinnen und -kandidaten, auch wenn sie von einer Gewerkschaft unterstützt werden, sind als Beschäftigte des Betriebes im Konfliktfall einem individuellen Druck ihres Arbeitgebers ausgesetzt. Dies schließt materielle Bevorzugung oder Benachteiligungen mit ein. Diese Personalisierung gibt dem Arbeitgeber Zugriffsmöglichkeiten, die ihm in tarifpolitischen Auseinandersetzungen mit der Gewerkschaft nicht zur Verfügung stehen (Bormann 2007, S. 17). Obwohl § 119 BetrVG die Behinderung der Wahl eines Betriebsrates ebenso wie die Benachteiligung oder Bevorzugung von Betriebsratsmitgliedern unter Strafe stellt, kommt es in der gerichtlichen Praxis nur äußerst selten zu entsprechenden Urteilen, wie Bormann am Beispiel mehrerer Konflikte bei Schlecker untersucht hat (ebd., S. 21). Nachdem sich 1994 Beschäftigte bei Schlecker über schlechte Arbeitsbedingungen und nicht tarifkonforme Löhne beschwert hatten, führte hier die spätere ver.di-Gründungsgewerkschaft HBV eine sechs Monate dauernde Kampagne durch, die schließlich im April 1995 in einen Tarifvertrag nach $\$ 3$ BetrVG mündete, in dem filialübergreifend 328 Betriebsratsbereiche definiert wurden, für die jeweils ein Betriebsrat gewählt werden konnte (Wohland 1995; Huhn 2001). Dies hielt allerdings das Unternehmen nicht davon ab, die Wahl von Betriebsratsgremien systematisch zu hintertreiben (Bormann 2007) und es somit den Ge- werkschaften schwer zu machen, die abgeschlossene Tarifvereinbarung mit Leben zu füllen. ${ }^{1}$ Andere prominente Beispiele für Betriebsratsvermeidung sind das schwedische Modehaus H \& M (Köhnen 2006), der Lebensmittelhändler Lidl sowie der Elektronikhändler Media Markt. Media Markt beschäftigte im Jahr 2011 etwa 17.000 Angestellte in 230 Filialen, von denen allerdings nur zwei über einen Betriebsrat verfügten. Während das Unternehmen argumentierte, diese geringe Zahl sei letztlich Ausdruck der besonders hohen Mitarbeiterzufriedenheit, beklagte sich die zuständige Gewerkschaft ver.di über die dort verbreitete Feindseligkeit gegenüber Gewerkschaft und betrieblicher Interessenvertretung, welche sich letztlich in systematischer Einschüchterung und Mobbing gegenüber betrieblichen Aktivistinnen und Aktivisten ausdrückte (Weber 2011). Obwohl die meisten dieser publik gewordenen Fälle aus dem Einzelhandel stammen, sind entsprechende Konflikte keineswegs auf diese Branche beschränkt (Bormann 2007, S. 11).

Allein anhand einzelner Berichte und Schilderungen lassen sich allerdings das Ausmaß und die Verbreitung betriebsratsfeindlichen Verhaltens des Managements nicht einschätzen. Zwar hat die Zahl der Medienberichte über entsprechende Ereignisse in den letzten Jahren zugenommen, jedoch lässt sich nicht sagen, ob dies tatsächlich auf die Zunahme entsprechender Aktivitäten oder eher auf eine gesteigerte Aufmerksamkeit der Medien zurückzuführen ist.

Wissenschaftliche Befunde über Managementmaßnahmen gegen Betriebsräte oder deren Wahl liegen kaum vor. Die letzte bekannte quantitative Analyse basiert auf einer Betriebsrätebefragung aus dem Jahre 1982. Nach dieser von Bormann zitierten Erhebung in einem DGB-Landesbezirk berichten $10 \%$ der befragten Betriebsräte von Versuchen des Arbeitgebers, Betriebsratswahlen zu beeinflussen. 3,5\% der Befragten sind darüber hinaus Fälle bekannt, in denen Arbeitgeber versucht haben, die Gründung eines Betriebsrates zu verhindern (Bormann 2007, S. 2 f.).

\section{Die WSI-Befragung}

Um diese Forschungslücke zu schließen, wurden mehrere DGB-Mitgliedsgewerkschaften angesprochen und um ihre Unterstützung für eine Befragung unter den Gewerkschaftssekretärinnen und -sekretären der jeweils untersten hauptamtlich besetzten Organisationsebene gebeten. Diese Grundeinheiten werden je nach Gewerkschaft als Bezirk, Verwaltungsstelle oder Region bezeichnet und haben

1 Bis zum Jahr 2000 konnten etwa 100 Betriebsräte gegründet werden (Dribbusch 2003); bis 2010 konnte diese Zahl auf 170 gesteigert werden. 
sehr unterschiedliche Größen und Ausdehnungen. Der Grundgedanke ist, über die jeweils für diese Grundeinheiten Verantwortlichen einen ersten Überblick über die Häufigkeit von gegen die Institution Betriebsrat gerichtetem Managementhandeln zu gewinnen. Wir nehmen an, dass solche Konflikte zumindest dann, wenn die Gewerkschaft in irgendeiner Form involviert war, den örtlich Verantwortlichen bekannt sein müssten. Wir unterscheiden zwei Konfliktbereiche: zum einen Maßnahmen, die darauf abzielen, die Errichtung von Betriebsräten zu verhindern, und zum anderen solche, die zum Ziel haben, bestehende Betriebsratsgremien aufzulösen oder in ihrem Handeln nachhaltig einzuschränken.

Vier Gewerkschaften, IG Metall, IG BCE, NGG sowie ver.di haben letztlich ihre Teilnahme an der Studie zugesagt. Während die Datenerhebung bei den drei erstgenannten Gewerkschaften relativ problemlos durchgeführt werden konnte, traf die Befragung im Organisationsbereich von ver. di auf einige strukturelle Hindernisse. Wegen der komplexen Organisationsstruktur war es bei ver.di, wie sich bald zeigte, nicht möglich, zuverlässige Auskünfte über die Grenzen der dreizehn Fachbereiche hinweg zu bekommen. Ein erster übergreifender Ansatz, bei dem die jeweiligen Bezirksgeschäftsführungen um Auskunft für ihren gesamten Organisationsbereich gebeten wurden, brachte keinen nennenswerten Rücklauf. Ersatzweise musste deshalb auf einzelne Fachbereiche zugegangen werden, wobei wir uns mit der Gewerkschaft darauf verständigten, unsere Erhebung zunächst lediglich im Fachbereich Handel durchzuführen, da hier auch die meisten Fälle vermutet wurden. Letztlich erwies sich dieser Ansatz als erfolgreich. Durch die Befragung in den Organisationsbereichen der drei Gewerkschaften und des ver.di FB Handel konnten Grundeinheiten, die insgesamt etwa $56 \%$ der Mitgliedschaft der DGBGewerkschaften repräsentieren, in die Erhebung einbezogen werden.

Der Fragebogen wurde von uns in Absprache mit den teilnehmenden Gewerkschaften entwickelt. Er wurde so kurz wie möglich konzipiert, um den Bearbeitungsaufwand in Grenzen zu halten und so die Rücklaufchancen zu erhöhen. In einem ersten Abschnitt wurden die örtlich verantwortlichen Gewerkschaftssekretärinnen und -sekretäre um Auskunft darüber gebeten, ob ihnen Fälle bekannt sind, bei denen der Arbeitgeber versucht hat, die Gründung/Wahl eines Betriebsrats zu verhindern. War dies der Fall, so wurden weitere Details zu diesen konkreten Fällen abgefragt. Um Ausfälle ebenso wie Befragungsabbrüche zu verringern, haben wir diese Nachfragen zu den einzelnen Fällen auf maximal drei Betriebe pro Gewerkschaftseinheit beschränkt. In einem zweiten Teil der Erhebung wurde nach gegen bereits existierende Betriebsratsgremien gerichtete Maßnahmen gefragt. Im Winter 2011/12 (im Fall von ver.di FB Handel im Winter 2012) wurde eine elektronische Fassung des Fragebogens an die jeweils zuständigen Gewerkschaftssekretäre der lokalen Organisationseinheiten (Bezirke, Regionen, Verwaltungsstellen) verschickt. Um Doppelbefragun- gen zu vermeiden, achteten wir sehr darauf, dass sich Organisationseinheiten nicht überschneiden. Insgesamt wurden 347 Fragebögen an die entsprechenden Bezirke (ver. di, IG BCE), Regionen (NGG) und Verwaltungsstellen (IG Metall) verschickt. Die Adressaten wurden gebeten, Auskunft über einschlägige Aktivitäten seit 2010 zu geben und die vorgelegten Fragen für ihren gesamten Zuständigkeitsbereich zu beantworten. Bis zum Ende der Befragung (Frühjahr 2013) konnten 184 gültige Fragebögen erfasst werden, was einer Rücklaufquote von 53 \% entspricht.

\section{Behinderung der Betriebsratswahl}

Insgesamt gaben $59 \%$ der Befragten an, dass ihnen für ihren Zuständigkeitsbereich Versuche der Be- oder Verhinderung einer Betriebsratswahl bekannt sind. $41 \%$ der Befragten antworteten, dass sie keine derartigen Fälle kennen. Allerdings bedeutet dies nicht notwendigerweise, dass es im Organisationsbereich dieser Bezirke, Regionen und Verwaltungsstellen zu keiner Wahlbehinderung gekommen ist, sondern zunächst einmal nur, dass den Befragten zumindest keine derartigen Fälle bekannt wurden.

Jene 59 \% der Befragten, die entsprechende Managementaktivitäten benannt haben, identifizierten insgesamt $241 \mathrm{Be}$ triebe, in denen der Arbeitgeber nach ihrer Kenntnis versucht hat, die Wahl eines Betriebsrates zu vereiteln. In etwa einem Drittel dieser Fälle (34 \%) war das Management mit seiner Strategie erfolgreich, d. h. die Wahl eines Betriebsrates ist letztlich gescheitert. Wie in Tabelle 1 gezeigt, ergeben sich hier einige Unterschiede, wenn man die vier in die Erhebung einbezogenen gewerkschaftlichen Organisationsbereiche miteinander vergleicht.

\section{TABELLE 1}

\section{Behinderung der Betriebsratswahl nach gewerkschaftlichem Organisationsbereich}

\begin{tabular}{lc|c}
\hline & $\begin{array}{c}\text { Versuche der Verhinderung der } \\
\text { Betriebsratswahl* }(\mathbf{n = 1 8 4 )} \\
\text { Angaben in Prozent der befragten } \\
\text { Gewerkschaftseinheiten }\end{array}$ & $\begin{array}{c}\text { Wenn bekannt, Anzahl der Fälle pro } \\
\text { lokale Gewerkschaftseinheit } \\
\text { (n=108) }\end{array}$ \\
\hline $\begin{array}{l}\text { ver.di } \\
\text { (FB Handel) }\end{array}$ & 66 & 2,9 \\
\hline NGG & 63 & 1,7 \\
\hline IG Metall & 56 & 2,2 \\
\hline IG BCE & 56 & 1,8 \\
\hline Insgesamt & 59 & $\mathbf{2 , 2}$ \\
\hline
\end{tabular}

*Dem Befragten ist mindestens ein Fall von Behinderung bekannt. Quelle: WSI-Befragung hauptamtlicher Gewerkschafter (2012). 
TABELLE 2

\section{Maßnahmen des Managements gegen die Wahl eines Betriebsrates}

Angaben in Prozent

\begin{tabular}{ll}
\hline Maßnahme & Anteil der Fälle* \\
\hline Einschüchterung möglicher Kandidaten für den Betriebsrat & 73 \\
\hline Verhinderung der Bestellung eines Wahlvorstandes & 43 \\
\hline Kündigung von Kandidaten für den Betriebsrat & 24 \\
\hline Kündigung von Mitgliedern des Wahlvorstandes & 18 \\
\hline “Herauskaufen” von Kandidaten & 16 \\
\hline Weigerung der Herausgabe von Personallisten & 12 \\
\hline Gezielte Reorganisation/Aufspaltung des Unternehmens & 5 \\
\hline Verlagerung, Ausgründung, Schließung des betreffenden Betriebes & 3 \\
\hline Sonstige & 25 \\
\hline
\end{tabular}

*Pro Fall können mehrere Maßnahmen angegeben werden.

Quelle: WSI-Befragung hauptamtlicher Gewerkschafter (2012).

Zunächst einmal fällt auf, dass Fälle der Behinderung von Betriebsratswahlen am häufigsten im Fachbereich Handel von ver.di vorkommen (66 \% der befragten Einheiten identifizierten mindestens einen Fall gegenüber $59 \%$ bei allen Gewerkschaften). Dies war zu erwarten, schließlich stammt eine Vielzahl der publik gewordenen Fälle aus dieser Branche. Der Eindruck bestätigt sich, betrachtet man die Anzahl der Fälle pro Gewerkschaftseinheit (hier wurden nur jene 108 lokalen Gewerkschaftseinheiten berücksichtigt, die überhaupt Aktivitäten aufwiesen). Auch hier hält der Handel die Spitzenposition: Sind überhaupt Versuche bekannt, die Wahl eines Betriebsrates zu behindern, so werden im Handel durchschnittlich 2,9 Fälle pro lokaler Gewerkschaftseinheit genannt, für die gesamte Umfrage beträgt dieser Durchschnitt 2,2 Fälle.

Weitere qualitative Erhebungen sind nötig, um die Unterschiede genauer zu verstehen und zu erklären. Erste stichprobenartige Nachfragen deuten darauf hin, dass es einen vermutlich starken Zusammenhang zwischen einer auf Expansion ausgerichteten gewerkschaftlichen Organisierungspraxis und dem Niveau der gegen die betriebliche Mitbestimmung gerichteten Managementaktivitäten gibt. Demnach steigt die Zahl der Fälle von Behinderung der Betriebsratswahl in jenen örtlichen Bereichen an, in denen sich Gewerkschaften aktiv und nachhaltig um die Gründung neuer Betriebsräte bemühen. Anders gewendet: Belassen es Gewerkschaften eher beim Status quo und zeigen keine oder nur geringe Initiativen zur Gründung von Betriebsräten, gibt es für das Management auch nur wenig Anlass, sich zu sträuben.

Weiterhin haben wir Gewerkschaftssekretäre um Auskunft darüber gebeten, welche konkreten Maßnahmen das Management zur Behinderung der Betriebsratswahl ergrif- fen hat. Weil wir die Detailinformationen pro Gewerkschaftseinheit auf maximal drei Betriebe beschränkt haben, dokumentieren wir im Folgenden die Ergebnisse für 191 der insgesamt 241 identifizierten Fälle.

Die verbreitetste Maßnahme zur Behinderung der Betriebsratswahl ist die Einschüchterung von Kandidatinnen und Kandidaten (Tabelle 2). Insbesondere in kleinen und mittelgroßen Betrieben, in denen der Kontakt zwischen Eigentümer, Management und Beschäftigten oftmals persönlich und direkt ist, lassen sich Kandidierende durch diese Taktik besonders leicht unter Druck setzen. Dieser Verdacht bestätigt sich insofern, als nach Angaben der Befragten $70 \%$ der Betriebe, für die Maßnahmen gegen die Wahl eines Betriebsrates dokumentiert wurden, weniger als 200 Personen beschäftigten. Darüber hinaus werden 60,1 \% dieser Betriebe durch den Inhaber selbst geführt.

Versuche der Wahlbehinderung beginnen zumeist recht früh im Prozess. So hat das Management in $43 \%$ der Fälle versucht, die Bestellung eines Wahlvorstandes zu verhindern, einem Gremium, das letztlich die Wahlen durchführt und überwacht. Sehr direkte und konfrontative Maßnahmen wie die Entlassung von Betriebsratskandidaten (24\%) oder von Mitgliedern des Wahlvorstandes (18\%) erwiesen sich ebenfalls als recht weit verbreitet. An dieser Stelle sei noch einmal daran erinnert, dass all diese Maßnahmen eine Verletzung der Vorschriften des Betriebsverfassungsgesetzes darstellen.

In der Mehrzahl der Betriebsfälle ergreift das Management lediglich eine oder zwei der in Tabelle 2 genannten Maßnahmen zugleich (bei Ausschluss der Kategorie „Sonstige“). Doch verbleiben nach Angaben der befragten Gewerkschaftssekretäre immerhin $35 \%$ der Betriebe, in denen Arbeitgeber drei und mehr Maßnahmen gleichzeitig anwenden, um letztlich die Wahl eines Betriebsrats zu verhindern.

Wenn wir die vier gewerkschaftlichen Organisationsbereiche miteinander vergleichen, ergeben sich einige bemerkenswerte Unterschiede: So ist die Einschüchterung von Kandidaten zur Betriebsratswahl mit 73 \% der Nennungen über alle Gewerkschaften hinweg die am häufigsten genannte Obstruktionstaktik; im Fachbereich Handel von ver.di ist diese Arbeitgebertaktik mit $86 \%$ aber überdurchschnittlich häufig vertreten. Ähnliches gilt für das „Herauskaufen“von Kandidaten, also die Gewährung von Vorteilen für den Fall, dass Beschäftigte von der Kandidatur zum Betriebsrat Abstand nehmen. Auch diese Maßnahme tritt im Handel deutlich häufiger auf als in allen anderen Organisationsbereichen, für die uns Daten vorliegen: Während „Herauskaufen“ im Handel in $21 \%$ der einschlägigen Fälle berichtet wird, beträgt dieser Anteil nur $16 \%$ im gesamten Datensatz. Spiegelbildlich verhalten sich die anderen Maßnahmenkategorien: Kündigung von Kandidaten ( $24 \%$ im gesamten $\mathrm{Da}$ tensatz, $12 \%$ im Handel) bzw. Wahlvorstandsmitgliedern (18\% alle, $2 \%$ Handel) oder die Verhinderung der Bestellung eines Wahlvorstandes ( $43 \%$ alle, $36 \%$ Handel). Diese Maßnahmen werden deutlich seltener genutzt als in den 
Organisationsbereichen von NGG, IG BCE und IG Metall.

Wir nehmen an, dass diese doch sehr deutlichen Branchenunterschiede letztlich Ausdruck der unterschiedlichen Arbeitskräftezusammensetzung in den einzelnen Organisationsbereichen sind. Während wir in der Metall- und in der chemischen Industrie von einem hohen Anteil männlicher, tariflich gesicherter Beschäftigter in Normalarbeitsverhältnissen ausgehen können, erweist sich insbesondere im Einzelhandel der Anteil von Frauen in atypischen Beschäftigungsverhältnissen als besonders hoch ${ }^{2}$. Zwar ist auch hier weitere Forschung notwendig, um das Verhältnis zwischen Geschlecht, Beschäftigungsform und Bezahlung auf der einen und Gegenmachtpotenzial auf der anderen Seite näher bestimmen zu können, es erscheint uns aber plausibel, davon auszugehen, dass Arbeitgeber im Prozess der Betriebsratsgründung mit schlecht geschützten Belegschaften anders umgehen als mit Normalarbeitnehmern. So sind finanzielle Angebote für Beschäftigte in Niedriglohnbereichen im Zweifelsfall besonders attraktiv, zumal wenn deren Annahme gegen die Aussicht möglicherweise lang anhaltender Schwierigkeiten abgewogen werden muss. Gleichzeitig sind die seitens der Arbeitgeber gezahlten Summen in diesen Fällen geringer als in ausgesprochenen Hochlohnbereichen. So müssen Arbeitgeber im Einzelhandel solange nicht auf direkt konfrontative Maßnahmen wie die Kündigung von Kandidaten und Wahlvorstandsmitgliedern, die im Zweifel auch sehr leicht kostspielige Gerichtsverfahren nach sich ziehen können, zurückgreifen, wie sie ihr Ziel bereits durch Einschüchterung oder die Gewährung von Vergünstigungen erreichen. Diese Logik einer Hierarchie von Einzelmaßnahmen deutet sich auch an, wenn wir uns nun der Frage zuwenden, inwieweit sich Arbeitgeber durch externe Unternehmensberatungen und Anwaltskanzleien unterstützen lassen.

Nach Angaben der befragten Gewerkschaftssekretäre haben sich Arbeitgeber in $43 \%$ der identifizierten Konfliktfälle bei ihren Versuchen, die Wahl eines Betriebsrates zu verhindern, durch Anwaltskanzleien oder Unternehmensberatungen unterstützen lassen. In weiteren 38 \% der Fälle konnte dies nicht zuverlässig eingeschätzt werden. Lediglich für $20 \%$ der Betriebe waren sich die Befragten sicher, dass keine dritte Partei im Spiel war. Nun galt Deutschland, anders als beispielsweise die USA oder das Vereinigte Königreich, bislang nicht als fruchtbares Betätigungsfeld für auf union busting spezialisierte Kanzleien (Behrens/Dribbusch 2013). Insofern Arbeitgeber in Personalfragen auf arbeitsrechtlichen Rat zurückgreifen mussten, konnten sie sich hier oft auf den entsprechenden Sachverstand der Arbeitgeberverbände stützen, die neben der Tarifpolitik auch arbeitsrechtliche Beratung und Prozessvertretung zu ihren Kernaufgaben zählen (Behrens 2011, S. 132f.). Seit einigen Jahren scheint hier einiges in Bewegung geraten zu sein: So bieten Kanzleien wie beispielsweise Naujoks aus Düsseldorf, aber auch einige andere, ihre Unterstützung bei der Bekämpfung betrieblicher Interessenvertretungen recht offensiv an (Esser/Schröder 2011, S. 46ff.). Unsere Befunde deuten bislang darauf hin, dass hier ein Markt für „Betriebsratsvermeidung" im Entstehen begriffen ist, doch erweist sich der „Marktanteil“ der einschlägig bekannten Kanzleien als noch sehr begrenzt. ${ }^{3}$ Wenn sich Arbeitgeber unterstützen lassen, so handelt es sich meist nicht um eine der wenigen spezialisierten Kanzleien, sondern eher um die jeweiligen örtlichen Hausanwälte. Aber auch das Gros der Hausanwälte macht einen Unterschied für die Praxis der Betriebsratsbekämpfung, wie eine genauere Analyse der Daten zeigt. Wenn wir jene Fälle aus der Analyse ausschließen, in denen unsere Auskunftspersonen schlichtweg nicht wissen, ob eine „dritte Partei“ involviert war, dann ergibt sich folgendes Bild: Die Wahrscheinlichkeit, dass ein Arbeitgeber drei oder mehr Maßnahmen der Wahlbehinderung gleichzeitig einsetzt, steigt um $10 \%$, wenn die befragten Gewerkschaftssekretäre angeben, dass eine Anwaltskanzlei oder Unternehmensberatung im Spiel war. Bemerkenswert erscheint weiterhin, dass im Bereich des ver.di-Fachbereichs Handel im Vergleich zum gesamten Datensatz deutlich seltener auf externe Unterstützung zurückgegriffen wurde: Während, bezogen auf alle in die Untersuchung einbezogenen Gewerkschaften, in $43 \%$ der Fälle eine externe Kanzlei/Beratung beteiligt war, beträgt dieser Anteil im Handel lediglich $26 \%$. Auch hier drängt sich der noch näher zu erforschende Verdacht auf, dass Arbeitgeber so lange nicht auf externe Unterstützung zurückgreifen (und diese teuer bezahlen) müssen, wie sie ihr Ziel auch mit einfacheren Mitteln erreichen können.

\section{Maßnahmen gegen bestehende Betriebsratsgremien}

Maßnahmen der Arbeitgeber, die das Ziel verfolgen, letztlich den Betriebsrat selbst oder einen Teil seiner Schlüsselpersonen loszuwerden, sind deutlich weniger verbreitet als Versuche, deren Wahl zu hintertreiben. So gaben lediglich $38 \%$ der befragten örtlichen Gewerkschaftsrepräsentanten an, dass es in ihrem Organisationsbereich überhaupt einen Versuch gegeben habe, Betriebsratsmitgliedern zu kündigen, das Gremium komplett aufzulösen oder Betriebsratsmitglieder zum Rücktritt zu bewegen (Tabelle 3).

2 Die Situation im Organisationsbereich der NGG ist hier weniger eindeutig: Während der Anteil gering bezahlter und atypischer Beschäftigung im Bereich von Beherbergungsgewerbe und Gastronomie besonders groß ist, kann hiervon in den klassischen Kernbereichen der Gewerkschaft wie Tabakwaren, Mühlen und Brauereien nicht die Rede sein.

3 In Fällen, in denen die befragten Gewerkschaftssekretäre angaben, eine dritte Partei sei involviert, wurde nach dem Namen der Kanzlei/Unternehmensberatung gefragt. 
TABELLE 3

Maßnahmen gegen gewählte Betriebsratsgremien nach gewerkschaftlichem Organisationsbereich

\begin{tabular}{l|c|c}
\hline & $\begin{array}{c}\text { Maßnahmen gegen gewählte } \\
\text { Betriebsratsgremien* }(\mathbf{n = 1 8 4 )} \\
\text { Angaben in Prozent der befragten } \\
\text { Gewerkschaftseinheiten }\end{array}$ & $\begin{array}{c}\text { Wenn bekannt, Anzahl der Fälle } \\
\text { pro lokale Gewerkschaftseinheit } \\
\text { (n=69) }\end{array}$ \\
\hline ver.di & 40 & 2,8 \\
\hline NGG & 47 & 1,3 \\
\hline IG Metall & 34 & 1,5 \\
\hline IG BCE & 41 & 1,3 \\
\hline Insgesamt & 38 & $\mathbf{1 , 7}$ \\
\hline
\end{tabular}

*Dem Befragten ist mindestens ein Fall mit Maßnahmen bekannt.

ABB.

\section{Arbeitgebermaßnahmen gegen betriebliche Mitbestimmung \\ Angaben in Prozent \\ - Behinderung der Betriebsratswahl Maßnahmen gegen existierenden Betriebsrat}

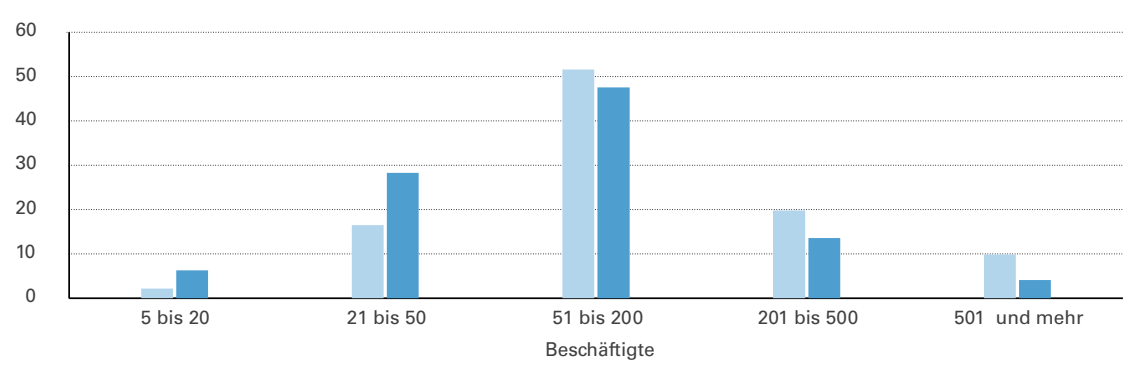

Vergleicht man diesen Anteil mit jenen 59 \% der Auskunftspersonen, die Versuche der Wahlverhinderung zu Protokoll gegeben haben, scheint sich das Management zumeist mit der Existenz des Betriebsrates - wenn es ihn erst einmal gibt - abgefunden zu haben, oder solche Versuche unterbleiben, weil sie letztlich zu beschwerlich oder kostenträchtig wären.

Wie Abbildung 1 zeigt, ergeben sich nur geringe Unterschiede bei der Betriebsgröße, vergleicht man jene Fälle, in denen Arbeitgeber die Gründung eines Betriebsrats verhindern wollen mit den Fällen, in denen Maßnahmen gegen bestehende Betriebsratsgremien ergriffen werden. In beiden Konstellationen fallen etwa $50 \%$ der Betriebe in die Größenklasse von 50 bis 200 Beschäftigte.

Da wir über keine belastbaren Angaben darüber verfügen, in wie vielen Fällen überhaupt die Gründung eines Betriebsrates in die Wege geleitet wurde, können wir keine
Aussagen darüber treffen, wie groß innerhalb der einzelnen Betriebsgrößenklassen die Wahrscheinlichkeit ist, dass Arbeitgeber die Gründung eines BR verhindern wollen. Wir interpretieren dennoch die präsentierten Befunde zur Größenverteilung dahin gehend, dass es eher die Belegschaften von kleinen und Mittelbetrieben sind, die zum Objekt mitbestimmungsfeindlicher Arbeitgeberpolitik werden.

Richten sich Arbeitgeber gegen bestehende Betriebsratsgremien, ist die häufigste Maßnahme die Kündigung einzelner Betriebsratsmitglieder (56 \% der Fälle, Tabelle 4).

In weiteren $35 \%$ der Fälle werden Betriebsratsmitglieder zum Rücktritt gedrängt und in $13 \%$ beantragen Arbeitgeber vor Gericht die Auflösung eines Betriebsrates. Gemäß $\$ 23$ BetrVG kann eine solche Auflösung vom Arbeitgeber oder einem Viertel der Belegschaft sowie der Gewerkschaft beantragt werden, wenn Fälle grober Pflichtverletzung nachgewiesen werden.

In $12 \%$ der Fälle versuchten Arbeitgeber den Betriebsrat auszuhebeln, indem sie den Betrieb in neue Einheiten aufgespalten haben. In extremen Situationen (3\%) schlossen Arbeitgeber gleich den gesamten Betrieb.

Auch im Fall der Maßnahmen gegen bestehende Gremien ergeben sich für den Handel einige Besonderheiten: Auch hier werden Maßnahmen der Einschüchterung („Betriebsrat zum Rücktritt gedrängt“) häufiger genutzt als bei den anderen Organisationsbereichen. ${ }^{4}$

Was nun betriebliche Schlüsselmerkmale anbelangt, fällt neben der Betriebsgröße wieder die Inhaberführung ins Auge. So macht es, bezogen auf die Stellung von Arbeitgebern zur Mitbestimmung, einen großen Unterschied, ob ein Betrieb durch die Eigentümer selbst oder durch ein angestelltes Management geleitet wird. In Tabelle 5 haben wir als Vergleichsmaßstab zunächst den Anteil der inhabergeführten Betriebe in der Gesamtpopulation dokumentiert (Spalte c).

Diese Berechnungen basieren auf Daten der WSI-Betriebsrätebefragung 2010, welche nur Betriebe aus der Privatwirtschaft mit Betriebsrat und mehr als 20 Beschäftigten berücksichtigt. Die Betriebsrätebefragung 2010 beinhaltet 1.984 gültige Fälle. Um die Vergleichbarkeit mit der hier zugrunde liegenden WSI-Befragung hauptamtlicher Gewerkschafter herzustellen, haben wir zwei Anpassungen vorgenommen: Erstens wurden in die Analyse nur solche Betriebe aufgenommen, die in den Organisationsbereich der drei beteiligten Gewerkschaften bzw. in das Branchencluster Handel fallen. Um einen möglichen Größenbias zu verringern, haben wir zweitens alle Betriebe mit mehr als $500 \mathrm{Be}-$ schäftigten ausgeschlossen. Nach diesen Anpassungen beträgt der Anteil inhabergeführter Betriebe $37 \%$, wobei dieser Anteil mit $45 \%$ bei der IG Metall am höchsten und im Organisationsbereich der IG BCE (30 \%) am geringsten ist.

4 Wegen der hier geringeren Fallzahlen sind die Unterschiede allerdings statistisch nicht sehr robust, sodass wir sie hier nicht im Detail dokumentieren. 
Wenn wir uns nun jenen 103 Betrieben zuwenden, für die Maßnahmen gegen bestehende Betriebsratsgremien berichtet wurden (Spalte b in Tabelle 5), so liegt hier der Anteil inhabergeführter Betriebe bei $49 \%$, also erstaunliche $16 \%$ über der Referenzkategorie. Innerhalb dieser zweiten Gruppe finden wir mit $22 \%$ inhabergeführter Betriebe den geringsten Anteil im Handel. Der Grund hierfür mag in der besonderen Netzwerkstruktur des Einzelhandels liegen. So wird der deutsche Einzelhandel durch Ketten wie ALDI, Lidl oder auch Metro dominiert, die jeweils über ein großes Filialnetz verfügen. Zwar werden die Ketten (Unternehmen) als Ganzes oft von den Eigentümerfamilien geführt, nicht aber die hier im Mittelpunkt stehenden Betriebe. Diese werden meist durch angestellte Filialleiter geleitet.

Wenden wir uns nun jenen 191 Betrieben zu, für die Versuche der Verhinderung der Wahl eines Betriebsrates berichtet wurden (Spalte a, Tabelle 5), so erreicht hier der Anteil inhabergeführter Betriebe $60 \%$.

Die präsentierten Befunde deuten an, dass Betriebsräte als eine der Kerninstitutionen der deutschen Arbeitsbeziehungen offenkundig dort auf verminderte Akzeptanz stoßen, wo Eigentümer die Geschäfte persönlich führen. In diesem Teil der deutschen Wirtschaft scheinen der „Herrim-Haus"-Standpunkt und eine geringe Bereitschaft, die betriebliche Entscheidungsgewalt zu teilen, besonders weit verbreitet zu sein. Dies bestätigen zum Teil ältere Befunde aus der Forschung zu Kleinbetrieben (Kotthoff/Reindl 1990; Hilbert/Sperling 1993). So beschreiben Kotthoff und Reindl paternalistische Orientierungen als Teil einer betrieblichen Sozialordnung. In unserem Kontext erweist sich die Inhaberführung letztlich als besonders aussagekräftige Variable, um die Segmentierung im Bereich der betrieblichen Mitbestimmung erklären zu können.

\section{Fazit}

Obwohl aktiver Arbeitgeberwiderstand gegen die Gründung von Betriebsräten ebenso wie aggressive Maßnahmen gegen bestehende Gremien bisher kein stilbildendes Merkmal der deutschen Arbeitsbeziehungen sind, so zeigen die Ergebnisse unserer Umfrage, dass es sich hierbei um mehr als eine vernachlässigbare Randerscheinung der deutschen Mitbestimmung handelt. Der wirkliche Umfang solcher mitbestimmungsfeindlicher Orientierungen und Strategien bleibt aber weiterhin schwer einzuschätzen. Insofern stellen die hier vorgestellten Befunde einen ersten Schritt in ein neues Forschungsfeld dar, dem aber noch weitere folgen müssten.

Immerhin kann als Anhaltspunkt, um die Verbreitung von Störungen der Betriebsratswahl ermessen zu können, folgende Information aus dem Organisationsbereich der IG Metall dienen: Demnach wurden bei den letzten tur-

\section{TABELLE 4}

\section{Maßnahmen, die gegen gewählte Betriebsratsgremien ergriffen wurden} Angaben in Prozent

\begin{tabular}{lc}
\hline Maßnahme & Anteil der Fälle* \\
\hline Kündigung von Betriebsratsmitgliedern & 56 \\
\hline Betriebsratsmitglieder zum Rücktritt gedrängt & 35 \\
\hline Beantragung der Auflösung des Betriebsrats beim Arbeitsgericht & 13 \\
\hline Gezielte Reorganisation/Aufspaltung des Unternehmens & 12 \\
\hline Verlagerung, Ausgründung, Schließung des betreffenden Betriebes & 3 \\
\hline Sonstige & 24 \\
\hline
\end{tabular}

* Pro Fall können mehrere Maßnahmen angegeben werden.

Quelle:WSI-Befragung hauptamtlicher Gewerkschafter (2012).

TABELLE 5

\section{Inhabergeführte Betriebe mit Behinderung der Betriebsratswahl bzw. des Betriebsrates}

Angaben in Prozent

\begin{tabular}{lc|c|c}
\hline & $\begin{array}{c}\text { Behinderung der } \\
\text { Betriebsratswahl } \\
(\mathbf{a})^{1} \\
(\mathbf{n}=\mathbf{1 9 1 )}\end{array}$ & $\begin{array}{c}\text { Maßnahmen gegen } \\
\text { bestehende } \\
\text { Betriebsratsgremien } \\
(\mathbf{b})^{1} \\
(\mathbf{n}=\mathbf{1 0 3})\end{array}$ & $\begin{array}{c}\text { Inhabergeführte } \\
\text { Betriebe insgesamt } \\
\text { (2010), Referenz- } \\
\text { gruppe } \\
\text { (c) })^{2}\end{array}$ \\
\hline ver.di (FB Handel) & 28 & 22 & 32 \\
\hline NGG & 56 & 55 & 38 \\
\hline IG Metall & 71 & 64 & 45 \\
\hline IG BCE & 75 & 38 & 30 \\
\hline Insgesamt & 60 & 49 & 37 \\
\hline
\end{tabular}

'Spalte (a) und (b): Angaben in Prozent der Fälle, in denen es zu Behinderungen der BR-Wahl/-Arbeit kam.

2 Spalte (c): Anteil aller inhabergeführten Betriebe im Organisationsbereich der jeweiligen Gewerkschaft.

Quelle: WSI-Befragung hauptamtlicher Gewerkschafter (2012); WSI-Betriebsrätebefragung (2010). nusmäßigen Wahlen von insgesamt 11.000 Betriebsratsgremien im Organisationsbereich der IG Metall immerhin 1.500 zum ersten Mal gewählt, allerdings ist nicht bekannt, in wie vielen dieser 1.500 Fälle es zu Problemen kam und wie viele Betriebsräte letztlich auf der Strecke blieben, weil sich mitbestimmungsfeindliche Arbeitgeber erfolgreich durchsetzen konnten. Wir sehen die Spitze eines Eisbergs, ohne zu wissen, wie groß die unter der Beobachtungsoberfläche liegende Masse ist. Ein großes Manko ist, dass uns bisher keine belastbaren Daten darüber bekannt sind, wie viele Fälle von Betriebsratsgründungen überhaupt jedes Jahr auftreten. Völlig im Dunkeln bleibt, in wie vielen Fällen Beschäftigte aufgrund eines mitbestimmungs- 
feindlichen Betriebsklimas eine Betriebsratsgründung gar nicht erst versuchen. Letztlich gibt es eine Reihe von Indizien dafür, dass gerade Arbeitgeber im Bereich der neu gegründeten Betriebe sowie in Branchen ohne traditionelle Verankerung der Schlüsselinstitutionen der Arbeitsbeziehungen Betriebsräten eher distanziert gegenüberstehen. Wie schwer Betriebsräte es haben, in solchen neuen Betrieben und Branchen Fuß zu fassen, zeigt schon das Beispiel der Solar- und Windenergie. Wir finden hier eine Reihe von Unternehmen mit zum Teil recht großen Produktionsstätten, die bislang keine oder aber eine recht schwache Verankerung der betrieblichen Mitbestimmung wie auch der Tarifbeziehungen aufweisen. Die Haltung des Managements gegenüber Betriebsräten und Gewerkschaften ist oft distanziert, teilweise sogar offen feindselig (Dribbusch 2013).

Wie unsere Befunde aber auch zeigen, erweisen sich die Betriebsgröße ebenso wie die Variable Inhaberführung als starke Indikatoren für feindliches Verhalten von Arbeitgebern gegenüber der betrieblichen Mitbestimmung. Und dies gilt über die Grenzen gewerkschaftlicher Organisationsbereiche hinweg.

Die weitere Bedeutung der hier analysierten Maßnahmen gegen Betriebsräte ergibt sich aus der besonderen Rolle der betrieblichen Mitbestimmung innerhalb des deutschen Systems der Arbeitsbeziehungen. Obwohl allein die Existenz eines Betriebsrates keine starke Gewerkschaftspräsenz im Betrieb zu garantieren vermag, lassen sich nur selten gut organisierte Belegschaften in betriebsratslosen Betrieben finden. Letztlich strahlen daher Versuche der Betriebsratsverhinderung weit über den betrieblichen Rahmen hinaus aus: Sie treffen die Fähigkeit der Gewerkschaften zur Reproduktion und Erneuerung ihrer Mitgliederbasis und auf diesem Weg auch die Funktionsfähigkeit der Tarifbeziehungen.

\section{LITERATUR}

Behrens, M. (2009): Still Married after All These Years? Union Organizing and the Role of Works Councils in German Industrial Relations, in: Industrial and Labor Relations Review 63 (3), S. 275-293

Behrens, M. (2011): Das Paradox der Arbeitgeberverbände. Von der Schwierigkeit, durchsetzungsstarke Unternehmensinteressen kollektiv zu vertreten, Berlin

Behrens, M./Dribbusch, H. (2013): Anti-unionism in a coordinated market economy: the case of Germany, in: Gall, G./Dundon, T. (Hrsg.): Global anti-unionism: nature, dynamics, trajectories and outcomes, Houndmills, S. 83-103 Böhm, S/Lücking, S. (2006): Orientierungsmuster des Managements in betriebsratslosen Betrieben. Zwischen Willkürherrschaft und Human Resource Management, in: Artus, I./ Böhm, S./Lücking, S./Trinczek, R. (Hrsg.): Betriebe ohne Betriebsrat. Informelle Interessenvertretung in Unternehmen, Frankfurt a. M./New York, S. 107-139

Bormann, S. (2007): Angriff auf die Mitbestimmung. Unternehmensstrategien gegen Betriebsräte - der Fall Schlecker, Berlin

Dribbusch, H. (2003): Gewerkschaftliche Mitgliedergewinnung im Dienstleistungssektor. Ein Drei-Länder-Vergleich im Einzelhandel, Berlin
Dribbusch, H. (2013): Nachhaltig Erneuern. Aufbau gewerkschaftlicher Interes senvertretung im Windanlagenbau, in: Wetzel, D. (Hrsg.): Organizing. Die Veränderung der gewerkschaftlichen Praxis durch das Prinzip Beteiligung, Hamburg, S. $92-118$

Ebbinghaus, B./Visser, J. (1997): When institutions matter: union growth and decline in Western Europe, 1950-90, ETUI-Draft-Paper, 15. Dezember, Brüssel Ellguth, P./Kohaut, S. (2013): Tarifbindung und betriebliche Interessenvertretung: Ergebnisse aus dem IAB-Betriebspanel 2012, in: WSI-Mitteilungen 66 (4), S. $281-288$, http://www.boeckler.de/wsi_43103_43110.htm

Esser, C./Schröder, A. (2011): Die Vollstrecker. Rausschmeißen, überwachen, manipulieren. Wer für Unternehmen Probleme löst, München

Greifenstein, R./Kißler, L./Lange, H. (2011): Trendreport Betriebsratswahlen 2010, HBS-Arbeitspapier 231, Düsseldorf, http://www.boeckler.de/pdf/ p_arbp_231.pdf (6.05.2013)

Haas, J. (2000): Ökonomischer, institutioneller, kultureller Wandel und gewerkschaftliche Mitgliedschaft 1968 bis 1993, Berlin

Hilbert, J./Sperling, H. J. (1993): Die kleine Fabrik. Beschäftigung, Technik und Arbeitsbeziehungen, München/Mehring

Huhn, J. (2001): Die Schlecker-Kampagne 1994-1995. Gewerkschaften als soziale Bewegung, Mannheim

Jirjahn, U. (2011): Ökonomische Wirkungen der Mitbestimmung in Deutschland. Ein Update, in: Schmollers Jahrbuch 131 (1), S. 3-57

Köhnen, H. (2006): Unternehmenskultur und Personalpolitik. Zur Situation der Beschäftigten und der Interessenvertretung bei H \& M, HBS-Arbeitspapier 119, Düsseldorf

Kotthoff, H./Reindl, J. (1990): Die soziale Welt kleiner Betriebe. Wirtschaften, Arbeiten und Leben im mittelständischen Industriebetrieb, Göttingen Küppers, K. (2012): Die Schlecker-Saga, in: die tageszeitung, 24/25.03.2012, S. $16-18$

Logan, J. (2006): The union avoidance industry in the United States, in: British Journal of Industrial Relations, 44 (4), S. 651-675

Schmidt, R./Trinczek, R. (1999): Der Betriebsrat als Akteur der industriellen Beziehungen, in: Müller-Jentsch, W. (Hrsg.): Konfliktpartnerschaft. Akteure und Institutionen der industriellen Beziehungen, München/Mering, S. 103-128 Weber, S. (2011): Betriebsratsfreie Zone, in: Süddeutsche Zeitung vom 17.02.2011

Wohland, U. (1995): Kampf um soziale Mindeststandards. 6 Monate Auseinandersetzung um die Drogeriekette Schlecker, in: Express. Zeitung für sozialistische Betriebs- und Gewerkschaftsarbeit, 33 (4), S. 9-10

\section{AUTOREN}

MARTIN BEHRENS, PD Dr., Wissenschaftler im Wirtschafts- und Sozialwissenschaftlichen Institut (WSI) in der Hans-Böckler-Stiftung. Arbeitsschwerpunkte: Vergleichende industrielle Beziehungen, Arbeitgeberverbände, betriebliche Mitbestimmung.

martin-behrens@boeckler.de

HEINER DRIBBUSCH, Dr., Wissenschaftler im WSI in der Hans-Böckler-Stiftung. Arbeitsschwerpunkte: Arbeitsbeziehungen und Gewerkschaftspolitik.

heiner-dribbusch@boeckler.de 